\title{
State Parameter Test Research of the Automatic Ramming Organ System
}

\author{
Guobao Ding $^{1, \text { a }}$, Rui Zhu ${ }^{2, b}$, He Cui ${ }^{3, \mathrm{c}}$ \\ ${ }^{1,2,3,4}$ Armor Technique Institute of PLA, Changchun ,China

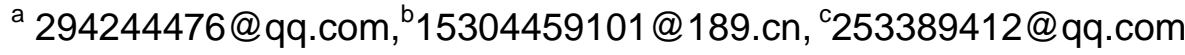

Keywords: the automatic ramming organ system; state parameter; LabVIEW

\begin{abstract}
Through analysis related signal ADMAS simulation during exercise of the feed mechanism,shell transfer arm,rammer of the automatic ramming organ system,set up an automatic ramming organ system state parameters test system, which is made up of a set of laser displacement sensor,encoder,angular velocity sensor,angle sensor,pressure sensor,data acquisition card.industrial computer and PC software.Design test system software based on LabVIEW.The system software includes test system calibration module,data acquisition module and historical data transferred and the data processing module.Dispatched by the main module of each sub-module,complete calibration test system,data collection.processing.Take use of related equipment to calibrate the test system.Conduct a series of test experiment for the automatic ramming organ system and get a part of accurate and reliable experimental data.
\end{abstract}

\section{Introduction}

Automatically for losing play system is a kind of mechanical and electrical integration of liquid system, such as reload, replenishment device can achieve mechanical part of the action, also can control agency action sequence control part and can provide dynamic hydraulic parts, is a very complex system. Link each other between different institutions, components, which makes automatic for lost play system uncertainties and concurrency, the failure characteristics of link of work and any a malfunction in complex structures will likely lead to severe fault, the whole gun weapon system and the automatic loading system is the highest rate of failure occurs in the artillery system, one of the good or bad will directly affect the performance of the power of artillery, the ability to survive. Therefore, the automatic fault prediction for lost play system diagnosis is imminent.

Based on this, this paper designs a set of the Angle sensor, laser displacement sensor, pressure sensor, photoelectric encoder, angular velocity sensor, data acquisition card and industrial test system of record for automatically lose signal system running status parameters, for automatic for losing playing system fault prediction diagnosis provides the premise condition and sufficient data to support.

\section{Test System Hardware Design}

Test system in general by the upper machine software, data acquisition part, control instruments and sensors. Design a test system before, first of all should be done according to the experience of the tester and the measured signal test system overall design, secondly analyses the characteristics of the object being measured each signal parameters, this paper will analyze the signal ADMAS simulation, provide the basis for selection of the sensor. Finally will test system involved in equipment search, according to the principle of classification and summary, according to the characteristics of signal and sensor, regulate the performance of instrument, data acquisition module, select the most appropriate equipment. Shall ensure that the designed test system that is able to complete tasks and meet the principle of economic efficiency.

This set of test system is mainly completed automatically for losing plays in the working process of the system test of the main signal. Play main signal for machine shot displacement and reducer cylinder chain sprocket spindle rotation Angle and angular velocity, the coordinator of coordination 
and flip Angle,angular velocity, spring machine's hydraulic system pressure.After the comprehensive consideration to complete test system overall design, hardware Test system block diagram is shown in Fig. 1.

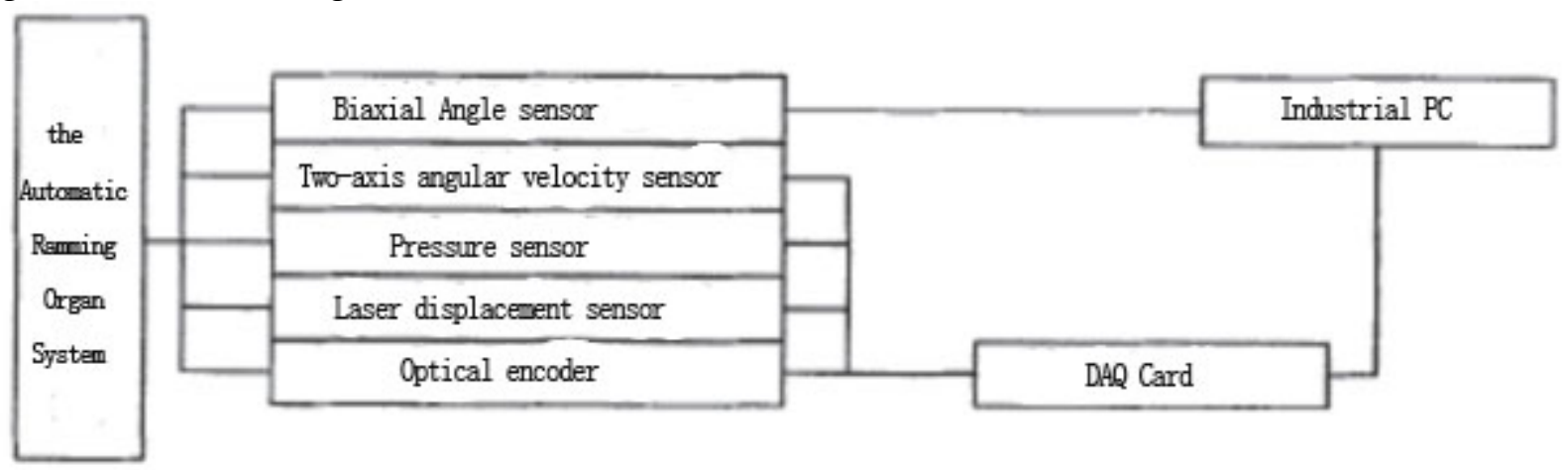

Fig. 1 test system block diagram

\section{Automatically for Lost Signal Analysis System and the Sensor Selection}

The maximum of large diameter howitzer firing rate about 10 rounds/min, you can see the entire automatic feed are not quickly lose play system run speed, thus automatically for lost play each state parameter signal frequency is not much, belong to the quasi static signal. But playing the system state parameters automatically for signals is different from general sports, in the process of their work has both smooth movement and transient motion, automatic for lost play system in the process of loading is always influenced by the vibration and shock. Sensors in the front of the testing system, direct contact with agency to be tested, the performance of the sensor of the test system has a decisive role, and sensor selection is the key of the test system design, the basic requirement of the test system of sensor are:

1) Due to mechanical automatic loading system structure is more complex, trouble to install sensors, consider ease of installation and lead. To improve the quality of sensor size, do more consideration.

2) The precision and high sensitivity, fast response.

3) have good stability, high signal-to-noise ratio, strong anti-jamming capability, safe and reliable, long life.

4) According to the simulation to determine the parameters such as signal amplitude ADMAS, ensure that the performance of the selected sensors to be redundant.

For the machine related signal analysis and sensor selection.

For spring machine is located in the rear turrets, mainly composed of several interconnected storage barrel barrel chain, the whole barrel chain under active combination sprocket drive can do the cycle of 360 degrees along the guide rail. Chain type TuiDan device mounted on the frame body. Is responsible for the choice of the projectile ammunition handling machine and using TuiDan editor projectile the pushed to the coordinator of the elastic plate. For the machine testing signals including barrel chain displacement and sprocket wheel spindle Angle and angular velocity in the reducer.Fig. 2 for the barrel chain ADMAS simulation displacement curve, the simulation curve, we find that the amplitude of about $370 \mathrm{~mm}$, larger displacement. The whole simulation time is $2.5 \mathrm{~s}$, $1.4 \mathrm{~s}$, before the barrel moving rapidly, after the basic no longer move. 


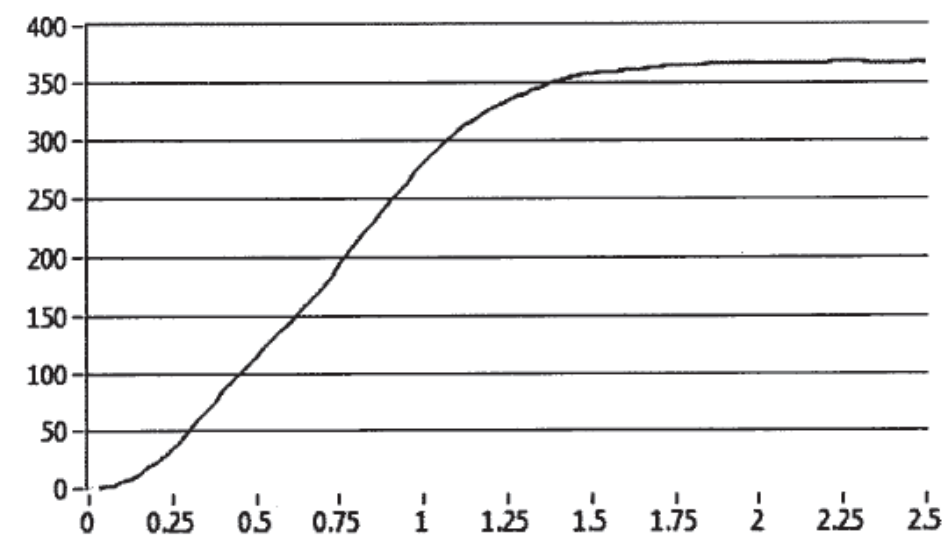

Fig. 2 barrel chain ADMAS simulation displacement curve

At present, the electrical and optical displacement sensor applied more widely. Electronic sensor signal is mainly to convert the displacement parameters for electric parameters. General electrical sensors, such as capacitance, inductance sensor, the eddy current sensor and so on, is comparatively mature technology, but the range is relatively small; Optical sensor research started late, such as laser displacement sensor, optical fiber displacement sensor, etc. Precise non-contact measurement of the position of the object to be tested, the change such as displacement, anti-interference ability is stronger, but the price is higher.

Due to play in the machine chain displacement measurement of rotary, displacement range is larger. Considering the ammunition handling machine structure is more complex, and the automatic feed lose play system works within the self-propelled guns, all kinds of interference is more, so it's best to choose the non-contact measurement, anti-interference ability strong optical displacement sensor. Optical displacement sensor are foreign companies on the research of the laser displacement sensor is mature, has become selection Keyence company IL600 laser displacement sensor, the main performance parameters are shown in Tab.1.

Tab.1 Laser displacement sensor main performance parameters

\begin{tabular}{|l|l|}
\hline Performance index & parameter \\
\hline Input(VDC) & $10 \sim 30$ \\
\hline reference distance(mm) & 600 \\
\hline measurement range(mm) & 800 \\
\hline Output(V) & $0 \sim 5$ \\
\hline repeated accuracy(um) & 50 \\
\hline degree of linearity & $\pm 0.5 \% \mathrm{~F} . S$ \\
\hline
\end{tabular}

ADMAS chain wheel spindle signal simulation curve as shown in Fig.3.Angle amplitude around 1800, after 1.5 s Angle change slowly. Angular velocity amplitude/s about 175 degrees, duration of amplitude around the ls. Chain wheel spindle after $2 \mathrm{~s}$ almost no longer around.

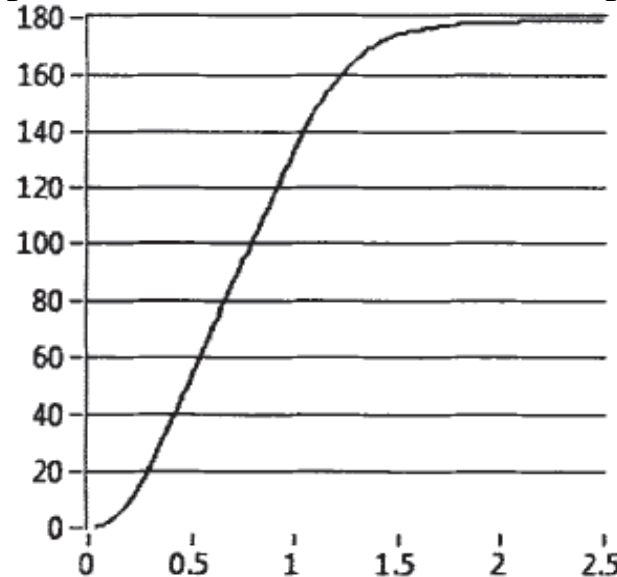

(a) rotation Angle

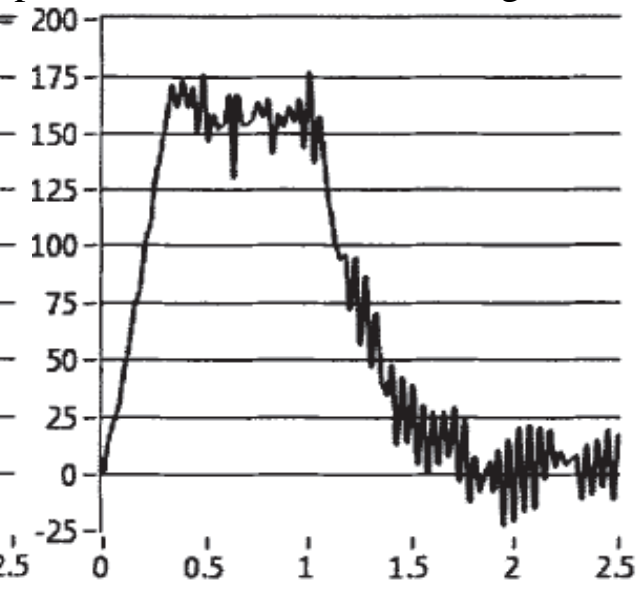

(b) rotation speed

Fig. 3 chain wheel spindle signal ADMAS simulation curve 
The coordinator related signal analysis and sensor selection.

For the machine after work, the coordinator to work. Coordinator work are divided into two steps: one is to coordinate the motor driver coordination arm around ear axis rotation coordinate to the axis of the projectile and the axis of the barrel in parallel; 2 it is to play the hydraulic system drives pendulum plays cylinder push play tray, to put it to lose spring machine to play online. Coordinator work has the characteristics of load change is big, poor working conditions, it can fast moving projectile coordinate and reach the designated position, directly affect the quality and effect of projectile loading. Coordinator main test signal coordination Angle, angular velocity, turning Angle and angular velocity. Coordinator to coordinate direction and reverse direction perpendicular, can choose biaxial Angle sensor and the angular velocity sensor.

Fig. 4 for the coordinator Angle ADMAS simulation curve, coordinated time about $0.6 \mathrm{~s}$, its amplitude is about 520. Turnover time of about $1 \mathrm{~s}$, its amplitude is about 360. Before $0.7 \mathrm{~s}$, Angle increases faster, after $0.7 \mathrm{~s}$, Angle changes no longer. Coordinate point rise faster than the pendulum Angle.

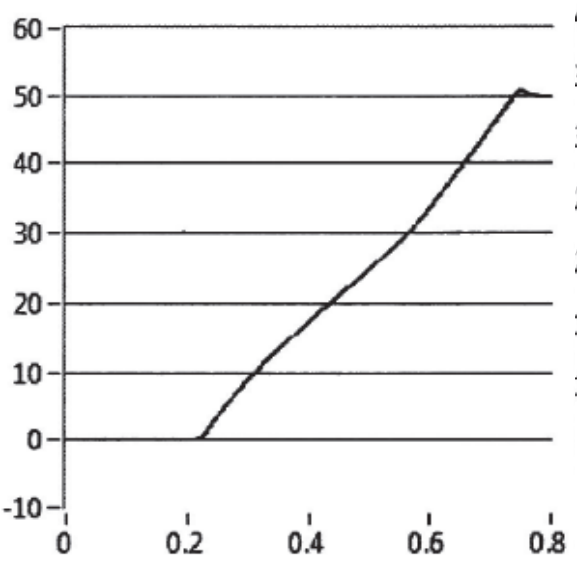

(a) coordination Angle

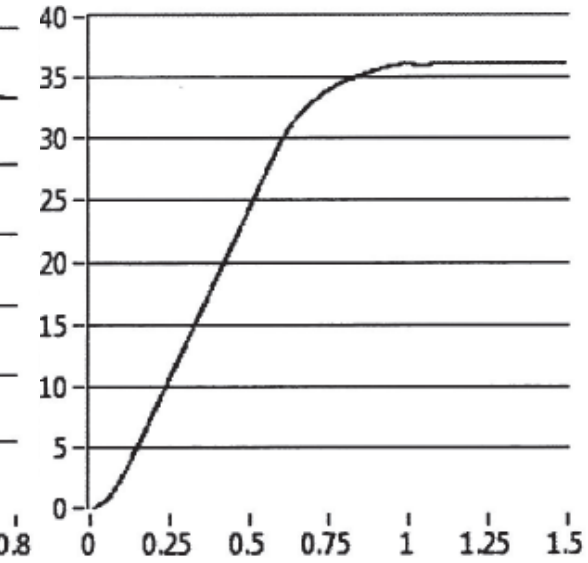

(b) flip Angle

Fig. 4 coordinator Angle ADMAS simulation curve

Now Angle sensor manufacturer is less, abroad such as Germany's Siemens, have xi 'an zte measurement and control, Beijing 7d aerial, etc. Final selection xi 'an zte tt\&c CS-2TAS-01B double shaft Angle sensor, the main parameters as shown in Tab.2.

Tab.2 Biaxial Angle sensor main performance parameter of CS-2TAS-01B

\begin{tabular}{|l|l|}
\hline Performance index & parameter \\
\hline measurement range & 75 \\
\hline accuracy & 0.5 \\
\hline distinguishability & 0.1 \\
\hline Digital interface type & RS232 \\
\hline
\end{tabular}

ADMAS coordinator angular velocity signal simulation, as shown in the Fig.5.Coordinate angular velocity amplitude after about 120 / s, began to coordinate, angular velocity quickly achieve amplitude. Compared with the coordination of angular velocity, turning angular velocity increase speed is much slower, about 450 / $\mathrm{s}$ amplitude. 

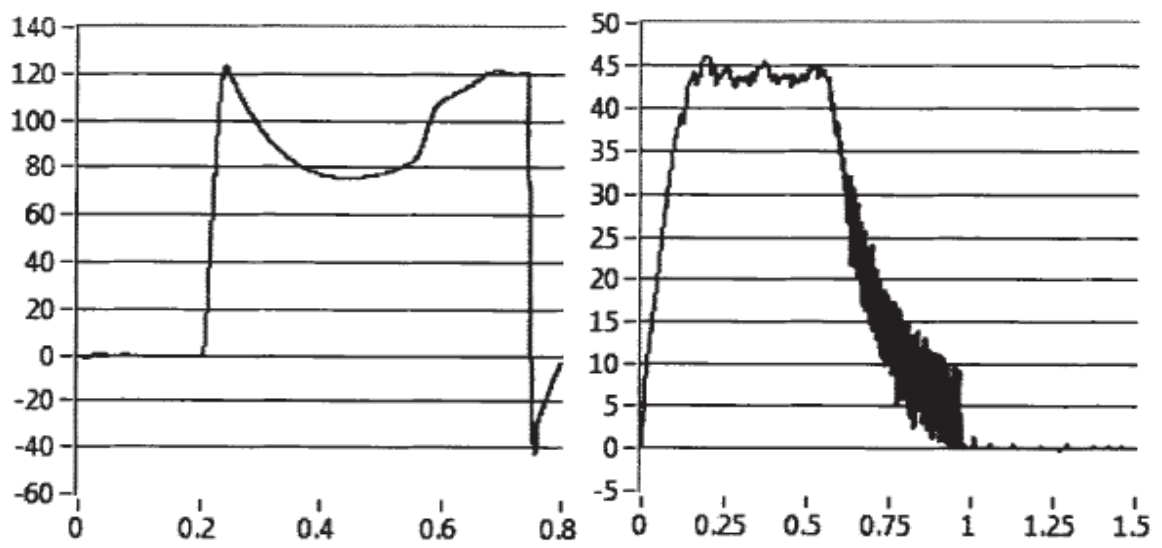

(a) coordination of angular velocity (b) flip angular velocity

Fig. 5 coordinator angular velocity ADMAS simulation curve

The angular velocity sensor is also known as a gyroscope. Gyroscope is commonly used in the form of the structure mechanical gyroscope, the optical gyroscope and micro mechanical gyroscope. Mechanical gyroscope, are dynamically tuned gyro and liquid floated gyro, flexible gyro and so on. Such gyroscope technology already quite mature, widely applied to weapons systems, such as Israel CONS artillery locating orientation system USES a K series developed by American beauty Lipton company dynamically tuned gyro, the bearing accuracy is less than 1 bit. But the traditional mechanical gyroscope shock and vibration resistance is poorer; Optical gyroscope, is widely used at the present stage mainly include optical fiber gyro and laser gyro. Fiber optic gyro, high impact resistance, strong accelerated motion resistance, long service life, in principle can be instant on, effectively overcome the blocking problem. Simple structure, small volume, light quality. Laser gyro: sTab. performance, strong anti-interference ability, wide dynamic range, is not sensitive to vibration and impact, and is not sensitive to cross shaft speed, start time is short, high reliability. But the laser light source and noise suppression of polarizer and zero drift need improvement, process complex, blocking phenomenon existed during low speed, high price. The detection sensitivity and resolution several orders of magnitude lower than the fiber optic gyroscope. Micro mechanical gyro, according to the principle of different, it can be divided into the elder brother's acceleration effect of micro vibration gyro, fluid gyroscope, micro gyroscope, suspended solid rotor gyroscope, micro gyroscope integrated optical gyroscope and atoms. Final selection as shown xi 'an zte tt\&c CS-2 ars two-axis angular rate sensor, the main parameters as shown in Tab.3.

Tab.3 Main performance parameters of the two-axis angular rate sensors of CS - 2 ars

\begin{tabular}{|l|l|}
\hline Performance index & parameter \\
\hline Input(VDC) & 5 \\
\hline incoming current(MA) & $\leq 20$ \\
\hline measurement range(。/s) & \pm 300 \\
\hline degree of nonlinearity $(\% \mathrm{FS})$ & $\leq 0.5$ \\
\hline Distinguishability $(\circ / \mathrm{s})$ & $\leq 0.04$ \\
\hline Bandwidty(HZ) & 100 \\
\hline Output(V) & $0.5-4.5$ \\
\hline
\end{tabular}

\section{Lose machines related signal analysis and sensor selection.}

Lose spring machine is responsible for the projectile loses to play online is fast and safe and reliable to the gun. Lose play machine by the hydraulic pressure drive, drive sprocket chain conveyor lose play online of the projectile, and with the artillery and part of the pitch, realize arbitrary Angle automatic loading. Lose play machine main test signal of the hydraulic system pressure.

Hydraulic ADMAS simulation curve is shown in Fig.6, in $0.2 \mathrm{~s}$ in maximum pressure reached around $12 \mathrm{MPa}$, duration of $0.05 \mathrm{~s}$, after a little ups and downs, there are two pressure $2.2 \mathrm{MPa}$ and 3.9MPa, respectively. 


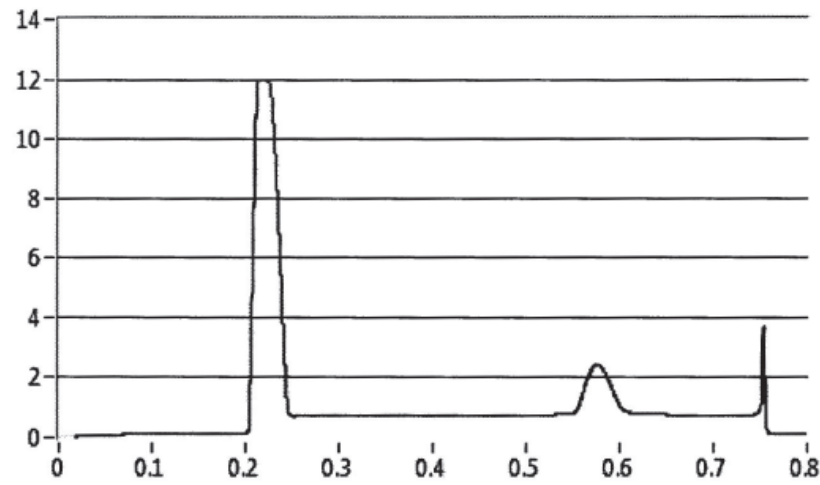

Fig.6 Hydraulic ADMAS simulation curve

Pressure sensor according to the principle of work can be divided into capacitive, strain type, hall, the resistance to pressure and piezoelectric, etc. Is more widely applied pressure resistance type and piezoelectric. Piezoresistive pressure sensor, relatively simple structure, high precision, high resolution, high frequency response, low power consumption, small size, high reliability. But more susceptible to the interference temperature piezoresistive sensor, prone to drift. Piezoelectric pressure sensor, good dynamic performance, high precision, usually for the transient pressure measurement. But the measurement of low frequency signal, prone to error and its output is charge need charge amplifier, high cost.

Considering the ammunition handling machine hydraulic system pressure signal frequency is not high, choose the piezoresistive pressure sensor. Piezoresistive pressure sensor main producers are currently abroad at endor, OMEGA companies in the United States, Germany Yi Fu door company, such as domestic Shanghai hengrui measurement and control, etc. Final selection Germany Yi Fu PN3001 pressure sensing door company.The main parameters as shown in Tab.5.

Tab5 PN3001 pressure sensor main performance parameter

\begin{tabular}{|l|l|}
\hline Performance index & parameter \\
\hline Input(VDC) & $18 \sim 36$ \\
\hline urement range (MPa) & $0-25$ \\
\hline repeatability (\% FS) & \pm 0.1 \\
\hline sluggish (\% FS ) & \pm 0.25 \\
\hline hing frequency (HZ) & 170 \\
\hline Output(V) & $0-10$ \\
\hline
\end{tabular}

\section{Test System Software Design}

The current commonly used PC software are mainly MFC programming, VB, VC, VC + +, LabVIEW, etc. Are the first four software text programming language, powerful, high efficiency and is suiTab. for various areas, but relatively complicated programming, started slowly, not only need to use programming language to realize various data processing methods, but also familiar with data communication protocols, so use this platform to develop test system software has certain difficulty, and the price is very low; The LabVIEW is a powerful the idea of data flow design of the graphical programming software, the original is designed to test and measurement. LabVIEW has the following advantages:

(1) simple, easy to learn. LabVIEW introduction soon, and do not like text language make a person feel very boring;

(2) open development environment. Through LabVIEW with third-party libraries and drivers to develop the required test system;

(3) the programming efficiency. LabVIEW programming efficiency is higher than the text language a lot, a few simple graphics and attachment will be able to complete the text language line dozens or even hundreds of lines of code to complete the function;

(4) data processing is simple and convenient. In the test system, the collected signal is not final purpose, generally need to undertake processing and analyzing, LabVIEW integrated with many 
function library and signal processing tools, data processing is very easy;

In the end, choose LabVIEW 2012 as system development software and driver software have NI company VISA and DAQNAVI research developed by the company.

Modularity is the basic characteristics of the software engineering, its core is the module hierarchy and independence. Modular reduces the complexity of software, greatly simplify the software design, debugging and maintenance, will not affect the whole by individual errors.

The whole test system is composed of software and hardware, software, including PC software and hardware drivers, and hardware driver is provided by the hardware manufacturers, including sensors, control circuit of the hardware and the data acquisition module. Although the precision of the test results depends on the hardware, but decided jointly by software and hardware reliability test system. Through software programming to realize the system hardware of the test drive control, complete the data transmission between computer and data acquisition module, as well as the data is stored and further processing. Test system software in test system and realize the interaction between the user operation function. The test system software design should meet the following requirements:

First, the software must have good man-machine interface, easy operation, ensure that the user can quickly familiar with the software operating steps. Second, the design process, as far as possible with modularization method. The advantages of this design approach is to ensure that each function module are independent of each other, if there is an error, can quickly identify where errors occur. Third, test software need interface reserved function expansion, to meet the needs of the software upgrade in the future. Fourth, safe, reliable, sTab. operation.

Test system software is mainly composed of test system calibration module, data acquisition module and the historical data of call and data processing modules. Sensors are used by module calibration; Data collection including sampling parameters setting and two parts, the parameter Settings including acquisition channel, sampling frequency and sampling time setting; Transferred to historical data and the data processing can be check on previous data and processing.

Module mainly complete test system calibration work. Its main working process is the use of standard instruments, sensor calibration of the corresponding work, get every job sensor sensitivity and error, for subsequent data processing parameters. Calibration module is divided into three parts: analog sensor, digital sensor calibration, RS232 sensor calibration. Calibration module data fitting with least squares method, and some positive and negative calibration schedule three times. First set parameters such as the channel setting, signal type, voltage range, sampling points, sampling rate, etc., in which the minimum value, increment, the maximum value is said to calibration of sensor standard input values, such as to PN3001 pressure sensor calibration Yi Fu door company, the minimum value, increment, the maximum set respectively $0,4,20$, said the calibration input values respectively $0,4,8,12,16,20 \mathrm{mpa}$. Parameter setting is completed, click on the button for sensor calibration, every click once completed the sampling, the sampling data in data display box, if there is something wrong with the acquisition of data, can be withdrawn through the button to remove the current value, and to collect. The whole calibration process is complete, click on the fitting calculation of static calibration results, and show in the interface to synthesis of straight line, according to the parameters of the fitting line of the slope and intercept, etc. Click "save" button, save the data, prepare for the back data processing.

\section{Test System Data Acquisition Module}

Acquisition module of the test system is collected automatically for transport system state parameters of electrical signals. For different types of sensors with different way of data collection. Laser displacement sensor, hydraulic pressure sensor, a two-axis angular rate sensor signal output is analog signal. Photoelectric encoder output signal to digital signal, and need to collect the output pulse signal of the frequency and quantity. For analog signal and digital signal acquisition method is through the data acquisition card to collect voltage signal. For xi 'an zte measurement and control of CS. 2 tas. $01 \mathrm{~b}$ double shaft Angle sensor, the signal output RS232 output, not through the data acquisition card and industrial computer on through the serial port acquisition signal. Data 
acquisition module for three types of sensors for collection at the same time.

\section{The RS - 232 data acquisition module.}

The RS - 232 is America's electronics industry alliance (EIA) published in 1969, the serial data communication interface standards. The RS - 232 standard stipulated in the logic 1 and logic 0 voltage series, transmission rate and the connector type. In RS - 232 standard, the character is a Serial transmission (Serial) way. Advantage is less transmission lines, wiring simple, the disadvantage is that the transmission distance up to $15 \mathrm{~m}$, this is due to the use of multi-core cable with $150 \mathrm{pF} / \mathrm{m}$ capacitance, and RS - 232 signal lines total load capacitance cannot exceed $2500 \mathrm{pF}$. The RS - 232 meet 1:3 for data transmission when maximum rate of $20000 \mathrm{~b} / \mathrm{s}$, transmission rate and transmission distance limit and reduced the communication distance can improve data transmission rate, and vice versa. Logic 1 negative level, between the $3 \mathrm{v}$ to $15 \mathrm{v}$, effective negative level signal state called mark, functional meaning to OFF. Logic 0 rules for level, between $+3 \mathrm{v} \sim+$ $15 \mathrm{v}$, is level signal state known as the space effectively, functional meaning to ON. Computer can use a serial port for data communication and data acquisition. Can set up their own communication protocol according to actual condition to complete the data transmission via a serial port.

Implemented in LabVIEW, RS - 232 data acquisition rely mainly on NI. VISA, NI. VISA is developed by the national instruments instrument bus to communicate with all kinds of advanced application programming interfaces (apis), it will not be restricted platform, bus, can on PXI, a serial port, GPIB, VXI, USB and Ethernet system configuration, programming. NI. VISA in LabVIEW as serial communication module, complete the instrument control and resource management, has been widely used.

\section{PCI1712 acquisition module.}

PCI1712 data acquisition module as shown in Fig.7 through DAQNavi Create Channel. The vi to choose the type of acquisition signals, such as analog input and output, digital input and output, the counter input and output, data acquisition and control card.

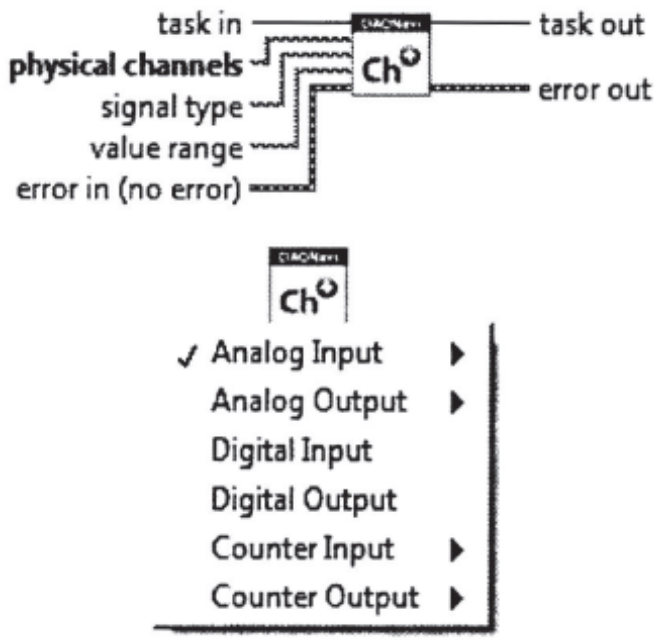

Fig.7 DAQNavi Create Channel.Vi

Historical data transferred to and the data processing module.

Historical data transferred to and the function of data processing module mainly has three aspects: one is already stored in the data in a file to read out, and be able to in the waveform display area; The second is the raw data collected according to different mathematical processing analysis to the test object, such as filter, add window, FFT transform. The third is combined with the sensitivity coefficient of sensor or after data fitting coefficient, converts the data after data processing analysis to the final quantities to test.

\section{Conclusion}

This article through to the automatic system for the lost shot for play machine, coordinator and lose machine play the analysis of the working process of the ADAMS dynamics simulation results, it is concluded that the measured signal parameters such as amplitude, and according to the 
characteristics of these parameters to complete the sensor selection, choose the data acquisition module and industrial PC, complete the design of the whole test system. In this paper, the design of sensor measurements can only by the upper machine software decoding and display, easy to watch, can consider to choose single chip microcomputer as controller and add LCD display, the implementation can be measured separately according to the Angle of the multi-function integrated sensor. Test system software can work normally, the reliability is high, but programming without considering the code efficiency, should also continue to optimize. Test system software should also continue to improve, plus some functions, such as reports, etc., make the software more comprehensive function

\section{References}

[1]M.Malesich,K.Fox.Overview and guidance for the DoD ATS Framework.IEEE AUTOTESTCON,2006:748.754.

[2]Droste.D.B,Allman.B.Anatomy of the next generation of ATE.Autotestcon,2005:560-569.

[3]Ross WA.The Impact of Next Generation Test Technology on Aviation Maintenance.IEEE AUTTOTESTCON,2003:2-9.

[4]Stora M J.Standard automatic test system(SATS)hardware interface Standards for SATS frameworks, VXI instrument front panels,power module interface,augmentation module interface, receiver fixture interface,pin map configuration.IEEE AUTOTESTCON,1998:627-638.

[5]Ramachandran N,Oblad $\mathrm{R}$ P.The role of a signal interface in supporting instrument interchangeability.IEEE AUTOTESTCON,2000:403-416.

[6]Mark Klafter,Diana Eshelman.Honeywell's Missiles Universal Set:An Automated,Windows-based,Test Station Providing Simultaneous System Testing Capability.IEEE Autotestcon,2003:11-18.

[7]S.Dhillonand,K.Chakrabarty.Sensor Placement for effective coverage and Surveillance in Distributed sensor networks.IEEE Wireless Communications and Networking Conference, 2003:1609-1614.

[8]Kang M H,Choi B W,Koh K C.Experimental study of a vehicle detector with an AMR sensor.Sensors and Actuators A 11 8,2005:278-284.

[9]JasonDing,Sing-YluCheung,Chin Woo Tan.Signal Processing of sensor Node Data For Vehicle Deteetion.2004 IEEE intelligent Transportation Systems Conference Washington.D.C.USA. 2004:3-6. 